\title{
ReaR

\section{Prácticas anestésicas seguras: ¿Sabemos que presión arterial intraoperatoria debemos mantener para evitar resultados postoperatorios desfavorables?}

Artículo original: Wesselink EM, Kappen TH, Torn HM, Slooter AJC, Van Klei WA. Intraoperative hypotension and the risk of postoperative adverse outcomes: a systematic review. British Journal of Anesthesia. May 2018: 1-16. (PubMed)

Caperote Sánchez L, Roca Trejo D, Macías Pingarrón JP, Bajo Pessini R

Complejo Hospitalario Universitario de Badajoz.

\section{Resumen}

La hipotensión intraoperatoria es un efecto secundario común de la anestesia general que puede producir una insuficiente perfusión de órganos. Sin embargo, no está clara la relación entre hipotensión durante cirugía no cardiaca y resultados desfavorables.

Métodos: Buscamos en PubMed, Embase, Web of Science y CINAHL, clasificando la calidad de los artículos analizados según los criterios STROBE y CONSORT. Las fuerzas de asociación de los estudios de alta calidad se clasificaron en riesgos de lesiones orgánicas específicas: insuficiencia renal aguda (IRA), lesión miocárdica, accidente cerebrovascular y lesiones orgánicas generales para diferentes umbrales de presión arterial.

Resultados: Presentamos una revisión de 42 artículos sobre las asociaciones entre varias definiciones de hipotensión intraoperatoria, y su relación con resultados postoperatorios desfavorables tras cirugía no cardíaca. Se informaron riesgos elevados de lesión orgánica por exposición prolongada ( $>10$ min) a presiones arteriales medias (PAM) $<80 \mathrm{mmHg}$ y para periodos más cortos $<70 \mathrm{mmHg}$. Los riesgos informados aumentan con la duración de las PAM $<65-60 \mathrm{mmHg}$ o para cualquier exposición con PAM $<55-50 \mathrm{mmHg}$.

Conclusiones: Las asociaciones reportadas sugieren que puede producirse lesión orgánica cuando disminuye la PAM $<80 \mathrm{mmHg}$ durante $>10 \mathrm{~min}$, y que este riesgo aumenta con la disminución progresiva de la presión arterial. Dado el diseño observacional retrospectivo de los estudios revisados, con gran variabilidad en las características del paciente, las definiciones de hipotensión y los resultados, no se pueden obtener conclusiones sólidas sobre qué presión arterial y bajo qué circunstancias son realmente peligrosas. Se ofrecen recomendaciones para el diseño de estudios futuros.

\section{Introducción}

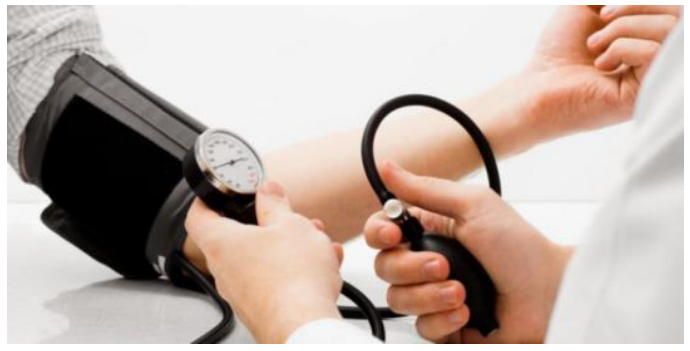

La hipotensión intraoperatoria es un efecto secundario común de la anestesia general que puede producir una insuficiente perfusión de órganos. Sin embargo, no está clara la relación entre hipotensión durante cirugía no cardiaca y resultados desfavorables.

Métodos: Buscamos en PubMed, Embase, Web of Science y CINAHL, clasificando la calidad de los artículos analizados según los criterios STROBE y CONSORT. Las fuerzas de asociación de los estudios de alta calidad se clasificaron en riesgos de lesiones orgánicas específicas: insuficiencia renal aguda (IRA), lesión miocárdica, 
accidente cerebrovascular y lesiones orgánicas generales para diferentes umbrales de presión arterial.

Resultados: Presentamos una revisión de 42 artículos sobre las asociaciones entre varias definiciones de hipotensión intraoperatoria, y su relación con resultados postoperatorios desfavorables tras cirugía no cardíaca. Se informaron riesgos elevados de lesión orgánica por exposición prolongada ( $>10 \mathrm{~min}) \mathrm{a}$ presiones arteriales medias $(\mathrm{PAM})<80$ mmHg y para periodos más cortos $<70$ mmHg. Los riesgos informados aumentan con la duración de las PAM $<65-60 \mathrm{mmHg}$ o para cualquier exposición con $\mathrm{PAM}<55-50 \mathrm{mmHg}$.

Conclusiones: Las asociaciones reportadas sugieren que puede producirse lesión orgánica cuando disminuye la PAM $<80 \mathrm{mmHg}$ durante $>10$ min, y que este riesgo aumenta con la disminución progresiva de la presión arterial. Dado el diseño observacional retrospectivo de los estudios revisados, con gran variabilidad en las características del paciente, las definiciones de hipotensión y los resultados, no se pueden obtener conclusiones sólidas sobre qué presión arterial y bajo qué circunstancias son realmente peligrosas. Se ofrecen recomendaciones para el diseño de estudios futuros.

\section{1.- Introducción}

La anestesia tiene un bajo riesgo específico, pero tiene un impacto importante en el riesgo perioperatorio, así como en los resultados. Los eventos adversos en el periodo perioperatorio siguen siendo frecuentes, sin embargo, un gran porcentaje de estos pueden prevenirse. Existen recomendaciones basadas en la evidencia científica y están disponibles para aumentar la seguridad del paciente. Las investigaciones futuras deberían proporcionar más evidencia de alta calidad sobre la efectividad de las prácticas seguras en los pacientes quirúrgicos y evitar aquellos eventos prevenibles como la hipotensión intraoperatoria.

La hipotensión intraoperatoria es un efecto adverso común en la anestesia general que ha recibido mucha atención en los últimos años debido a su elevada frecuencia y sus consecuencias adversas. Aunque no hay una definición ampliamente aceptada de hipotensión intraoperatoria, se están realizando estudios que analizan la asociación entre la hipotensión intraoperatoria y los resultados adversos postoperatorios. Para los anestesiólogos es esencial conocer de manera clara los efectos de la hipotensión intraoperatoria en la disfunción postoperatoria del órgano y la mortalidad, así como determinar el rango de tensión arterial aceptable durante la cirugía. En estudios observacionales recientes ya se ha demostrado la asociación entre la hipotensión y la insuficiencia renal aguda $o$ la lesión miocárdica, principalmente el infarto agudo de miocardio (1).

\section{2.- Descripción}

Se realizó una búsqueda sistemática en PubMed, Embase, Web of Science y CINAHL. De todas las publicaciones seleccionadas se obtuvieron datos sobre el diseño del estudio, las definiciones de hipotensión, los resultados estudiados y sus fuerzas de asociación. Además, se clasificó la calidad de los artículos analizados según los criterios STROBE y CONSORT adaptados. Tras la selección de artículos, la exclusión de aquellos no válidos y el análisis en profundidad de los restantes, se incluyeron 42 trabajos publicados entre 2002 y 2017 para la extracción de datos y evaluación de la calidad. El número de pacientes en los 42 estudios incluidos 
varió de 40 a 152.445 , con una mediana de 1.523 en cada estudio.

Existe una gran variabilidad en la definición de hipotensión en los distintos estudios analizados:

- Umbral basado en presiones sanguíneas absolutas.

- Presión arterial media (29 estudios)

- Presión arterial sistólica (17 estudios)

- Umbral basado en descenso relativo de la presión: disminución porcentual o absoluta en relación a la presión arterial basal.

- Presión arterial media (9 estudios)

- Presión arterial sistólica (7 estudios)

También existe gran variación en la información sobre la duración de la hipotensión. En 7 estudios se incluyó la duración mínima de la hipotensión en su definición. En el resto, sin embargo, se analizó la duración de dicha hipotensión con diferentes estrategias: minutos en los que la presión se encontraba por debajo del umbral, área bajo la curva de la presión umbral, por ponderación de tiempo, etc. En sus conclusiones, 14 estudios informaron sobre asociaciones con una duración mínima de hipotensión $\geq 1$ minuto, 8 estudios $\geq$ a 5 min, 12 estudios $\geq 10$ minutos y 7 estudios $\geq 20$ minutos.

Después de clasificar los estudios incluidos según el umbral de presión arterial utilizada, la calidad y los resultados estudiados, se comparó la fuerza de la asociación entre dichos umbrales y los resultados. Se clasificaron, para diferentes umbrales de presión arterial, en riesgos de lesiones orgánicas específicas: mortalidad, insuficiencia renal aguda, lesión miocárdica, accidente cerebrovascular, delirio y daño orgánico general. Las fuerzas de asociación se establecieron según el siguiente criterio:

- Fuerza de asociación de alto riesgo: $\mathrm{OR} / \mathrm{RR} / \mathrm{HR} \geq 2$

- Fuerza de asociación de riesgo moderado: OR/RR/HR 1,4 - 2

- Fuerza de asociación de bajo riesgo: OR/RR/HR 1 - 1,4

Una vez que un umbral específico de PAM y la duración de la hipotensión alcanzaron una clasificación de riesgo, esa clasificación se traspasó a todos los umbrales de PAM inferiores o a las duraciones de hipotensión más largas en el mismo umbral de PAM. Esto refleja la suposición de que las presiones sanguíneas más bajas o los episodios de hipotensión intraoperatoria más largos siempre agravan el riesgo de lesión orgánica.

Los resultados que se objetivaron fueron los siguientes:

- Mortalidad (analizada en 14 estudios): Incidencia 0,03-5.6\%

- Insuficiencia renal aguda (12 estudios): Incidencia 2,8-72\%

- Lesión miocárdica (9 estudios): Incidencia 0,09-30\%

- Accidente cerebrovascular (4 estudios): Incidencia 0,004-0,09\%

- Delirio (5 estudios): Incidencia 9-33\%

- Lesión orgánica general (5 estudios): Incidencia 29,7-37\% 
Los riesgos reportados de cualquier lesión orgánica después de cirugía no cardiaca aumentaron con la exposición prolongada y con presiones arteriales más bajas. Los resultados obtenidos fueron:

* Riesgo levemente aumentado:

$>$ Presión arterial media $<80$ $\mathrm{mmHg}$ durante $\geq 10$ minutos

$>$ Presión arterial media $<70$ mmHg durante $<10$ minutos * Riesgo moderadamente
aumentado:

$>$ Presión arterial media $<65-60$ mmHg durante $\geq 5$ minutos

$>$ Presión arterial media $<55-50$ $\mathrm{mmHg}$ durante cualquier duración

* Riesgos muy aumentados:

$>$ Presión arterial media $<65$ mmHg durante $\geq 20$ minutos

$>$ Presión arterial media $<50$ mmHg durante $\geq 5$ minutos

$>$ Presión arterial media $<40$ mmHg durante cualquier duración

Para insuficiencia renal aguda y lesión miocárdica, los riesgos comenzaron en umbrales $<65 \mathrm{mmHg}$ y aumentaron con el grado y la duración de la hipotensión. En relación a la mortalidad, se informó asociación con umbrales más altos que insuficiencia renal aguda y lesión miocárdica, comenzando cuando hubo exposición prolongada a PAM $<80$ $\mathrm{mmHg}$. Para ictus isquémico, solo fueron reportadas pequeñas fuerzas de asociación no significativas. Para el delirio, se encontraron asociaciones no significativas para una duración de PAM $<50 \mathrm{mmHg}$. Para lesión orgánica general, los datos obtenidos fueron insuficientes.

\section{3.- Análisis crítico}

El estudio que presentamos expone las conclusiones obtenidas tras la revisión conjunta de un importante número de estudios publicados en relación a los eventos adversos perioperatorios que pueden estar en relación con la hipotensión. Finalmente se analizaron en profundidad 42 artículos publicados en diferentes países, con un número de pacientes en cada uno de ellos entre 40 y 152.445 , lo cual hace que la validez externa de la revisión pudiese ser elevada.

Sin embargo al igual que los autores originales, creemos que la interpretación y aplicación clínica de los resultados de esta revisión se ven obstaculizadas por las grandes diferencias entre los estudios y las asociaciones observadas. Difieren sustancialmente en la selección de los grupos de pacientes o los procedimientos, en qué paciente y qué características del procedimiento fueron o no reportadas por los diferentes estudios y en la forma en que se definió y analizó la hipotensión intraoperatoria (2). También hubo una variación importante en la definición y en el análisis de los resultados postoperatorios adversos. Se informaron seis grupos diferentes de reacciones adversas postoperatorias: mortalidad, insuficiencia renal aguda, lesión miocárdica, accidente cerebrovascular isquémico, delirio y lesión orgánica general. Sin embargo, estos resultados tampoco fueron homogéneos sino que dentro de cada uno de ellos, a su vez, fueron estudiados diferentes resultados adversos con diferentes definiciones. Por ejemplo, la definición de lesión miocárdica varió desde biomarcadores elevados con o sin cambios en el ECG a complicaciones cardiovasculares según 
el estudio que consultemos, así como la definición de insuficiencia renal aguda, que incluyó desde alteraciones en las cifras de creatinina (en diferentes rangos de severidad) a alteración en el ritmo de diuresis: oliguria-anuria.

La gran heterogeneidad en las características iniciales, las definiciones de hipotensión $y$ estos resultados estudiados a los que hacemos referencia en el párrafo anterior, han hecho que para los autores fuese un desafío llegar a un resumen cuantitativo de los resultados. Por ello realizaron conversiones y suposiciones para poder fusionar las distintas definiciones de hipotensión intraoperatoria y poder informar sobre las fuerzas de asociación de los diferentes parámetros estudiados con la hipotensión.

Sin embargo, desde nuestro punto de vista, esta gran variabilidad en las distintas definiciones de hipotensión y de los eventos adversos analizados, así como la gran variabilidad en las características de los pacientes incluidos en el estudio (tipo de intervención, edad, patologías previas, etc) hacen que la validez interna y externa de este análisis pueda ser cuestionada y pueda verse reducida. Así, por ejemplo, en los resultados obtenidos, llama la atención el amplio intervalo de mortalidad, desde un 0.03 a un $5.6 \%$, lo cual puede ser una muestra de este posible sesgo de selección de artículos. Llama la atención también cómo se establecieron las fuerzas de asociación entre la hipotensión y los resultados adversos, dado que directamente se hizo siguiendo un criterio creado por los autores directamente: alto riesgo $(\mathrm{OR} / \mathrm{RR} / \mathrm{HR} \geq 2)$, riesgo moderado (OR/RR/HR 1,4 - 2) y bajo riesgo (OR/RR/HR 1 - 1,4).

Por estos motivos expuestos, a pesar de este análisis exhaustivo realizado, todavía es difícil definir de manera fiable un punto de corte para el cual la TA (tensión arterial) es demasiado baja. Ejemplo de ello es la gran heterogenicidad en los umbrales de TA bajo los cuales se empezaba a considerar que el paciente tenía hipotensión en cada uno de los estudios analizados. Así, algunos definían la hipotensión en base a la TAM (tensión arterial media) y otros en base a la TAS (tensión arterial sistólica), lo que deriva claramente, como hemos referido anteriormente, en una dificultad para el análisis de los resultados obtenidos $\mathrm{y}$, en consecuencia, una disminución de la validez interna del metaanálisis. A pesar de todo ello, sí parece claro que el riesgo de la lesión del órgano final parece aumentar rápidamente con la exposición prolongada a presiones sanguíneas intraoperatorias más bajas, según la evidencia actual, no se puede probar una relación causal directa entre las presiones sanguíneas intraoperatorias y los resultados finales.

Los autores del metaanálisis también concluyen que todos los factores e interacciones que contribuyen a la aparición de estos resultados adversos son difíciles de desentrañar $y$ discriminar y parece poco probable que podamos analizar la contribución de cada uno de estos factores por separados utilizando solamente datos de observación. Por estos motivos creemos que son necesarios estudios de intervención para comprender la relación causal entre TA baja intraoperatoria y resultados adversos. Los futuros estudios sobre la hipotensión intraoperatoria deberían establecer umbrales de TA claros dentro de grupos de pacientes específicos y para resultados específicos, perfectamente establecidos y definidos. Además, los autores originales proponen que éstos deberían incluir y analizar otras variables que son indicativas de causas subyacentes $y$ mecanismos de hipotensión, como la 
frecuencia cardíaca, variabilidad de presión de pulso (VVP), gasto cardiaco (GC) estimado mediante técnicas avanzadas y biomarcadores específicos. Esto nos permitiría estudiar hipótesis sobre los propios mecanismos de hipotensión en función del contexto y si las intervenciones específicas sobre ese mecanismo podría mejorar los resultados obtenidos.

Tras la publicación de esta revisión sistemática, también se han publicado otros artículos relacionados con este mismo tema. En un estudio de junio de 2018 ( $\mathrm{n}=$ 8782) (3), se analizó la relación entre hipotensión $\mathrm{y}$ morbimortalidad hospitalaria en pacientes sépticos en UCI. Los resultados indicaron que con $\mathrm{PAM}<65$ $\mathrm{mmHg}$ las probabilidades de mortalidad hospitalaria aumentaron 11.4\% (IC 95\% $7.8-15.1 \%$; $\mathrm{p}<0.001)$; las de lesión renal aguda aumentaron $7.0 \%$ (4.7$9.5 \% ; \mathrm{p}<0.001) ; \mathrm{y}$ las de lesión miocárdica aumentaron $4.5 \% \quad(0.4-$ $8.7 \% ; \mathrm{p}=0.03)$. Por ello, concluyeron que en pacientes sépticos en UCI (unidad de cuidados intensivos) se debe mantener PAM $>65 \mathrm{mmHg}$.

El estudio VISION ( $\mathrm{n}=15109)$ (4), también publicado en junio de 2018, investigó la relación entre cambios cardiovasculares intraoperatorios y efectos adversos. En relación a la hipotensión: PAS $<100 \mathrm{~mm} \mathrm{Hg}$ se asoció con lesión miocárdica (OR, 1,21 [1.05-1.39]; $\mathrm{P}=0.01$ ) y aumento de la mortalidad (OR, 1.81 [1.39-2.37]; P $<.01)$ mientras que PAS $>160 \mathrm{~mm} \mathrm{Hg}$ se asoció con lesión miocárdica (OR, 1.16 [1.01-1.34]; $\mathrm{P}=0.04)$ e infarto de miocardio (OR, 1.34 [1.09-1.64]; $\mathrm{P}=$ $.01)$.

Otro artículo $(\mathrm{n}=669)$ (5) publicado en julio de 2018, volvió a relacionar la lesión renal aguda con la hipotensión intraoperatoria, definida como PAM < $70 \mathrm{~mm} \mathrm{Hg}$ durante más de 5 minutos, de forma muy llamativa $(\mathrm{OR}=12.713$ [7.762-20.823]; $\mathrm{P}<.001)$. En resumen, existen numerosos estudios, tanto recientes como antiguos, que apuntan a que existe una relación clara entre hipotensión arterial y eventos adversos tales como la insuficiencia renal aguda, infarto agudo de miocardio o delirio, por ejemplo. Sin embargo, aunque todas estas relaciones no han podido quedar demostradas de forma significativa, probablemente por la falta de ensayos clínicos aleatorizados con amplio número de pacientes, debemos tenerlo en cuenta en nuestra práctica clínica habitual, dado que publicaciones actuales ya demuestran que algunas de estas relaciones son estadísticamente muy significativas.

\section{4.- Conclusiones}

Las asociaciones reportadas en esta publicación sugieren que el daño orgánico puede ocurrir cuando la presión arterial media disminuye $<80$ mmHg durante 10 min o más, y que este riesgo aumenta cuando las TA van siendo cada vez más bajas. Dado que la mayoría de los estudios son observacionales y retrospectivos, reflejan una gran variabilidad en las características del paciente, definiciones de hipotensión y resultados, no se pueden dictar conclusiones sólidas sobre cuál es el valor umbral de TA. Se necesitan ensayos de intervención prospectivos en grupos específicos de pacientes y realizar un análisis exhaustivo de resultados adversos para avanzar y poder desentrañar las dudas que aún podemos plantearnos sobre este tema.

\section{Bibliografía}

1. Walsh M, Devereaux PJ, Garg AX, Kurz A, Turan A, Rodseth RN et al. Relationship between intraoperative mean arterial pressure and clinical outcomes after noncardiac surgery: toward an empirical definition of 
hypotension. 2013;119:507-515. (PubMed) ( $\underline{\text { HTML) }}$

2. Vernooij LM, van Klei WA, Machina M, Pasma W, Beattie WS, et al. Different methods of modelling intraoperative hypotension and their association with postoperative complications in patients undergoing non-cardiac surgery. $\mathrm{Br} \mathrm{J}$ Anaesth. 18;120:1080-1089. (PubMed)

3. Maheshwari K, Nathanson BH, Munson SH, Khangulov V, Stevens M, Badani $\mathrm{H}$, et al. The relationship between ICU hypotension and inhospital mortality and morbidity in septic patients. Intensive Care Med (2018) 44:857-867. (PubMed) (HTML) ( $\underline{\mathrm{PDF}})$

4. Yu J, Park HK, Kwon HJ, Lee J, Hwang JH, Kim HY, et al. Risk factors for acute kidney injury after percutaneous nephrolithotomy. Implications of intraoperative hypotension. Medicine (Baltimore). 2018 Jul; 97(30):e11580. (PubMed) (HTML) (PDF)
5. Abbott TEF, Pearse RM, Archbold RA, Ahmad T, Niebrzegowska E, Wragg A. A Prospective International Multicentre Cohort Study of Intraoperative Heart Rate and Systolic Blood Pressure and Myocardial Injury After Noncardiac Surgery: Results of the VISION Study. Anesth Analg. 2018 Jun; 126(6):1936-1945. ( $\underline{\text { PubMed }}$ ( $\underline{\text { HTML) }}$ ( $\underline{\text { PDF }})$

Correspondencia al autor

Laura Caperote Sánchez

lauricapsan@hotmail.es MIR Anestesiología y Reanimación. Servicio de Anestesiología y Reanimación.

Complejo Hospitalario Universitario de Badajoz.

Aceptado para el blog en enero de 2019 